\title{
On the orbital structure of the HD 82943 multi-planet system
}

\author{
Roman V. Baluev ${ }^{1,2}$ and Cristian Beaugé ${ }^{3}$ \\ ${ }^{1}$ Central Astronomical Observatory at Pulkovo of Russian Academy of Sciences, Pulkovskoje \\ shosse 65, St Petersburg 196140, Russia \\ ${ }^{2}$ Sobolev Astronomical Institute, St Petersburg State University, Universitetskij prospekt 28, \\ Petrodvorets, St Petersburg 198504, Russia \\ email: r.baluev@spbu.ru \\ ${ }^{3}$ Instituto de Astronomía Teórica y Experimental, Observatorio Astronómico, Universidad \\ Nacional de Córdoba, \\ Laprida 854, (X5000BGR) Córdoba, Argentina
}

\begin{abstract}
HD 82943 hosts a mysterious multi-planet system in the 2:1 mean-motion resonance that puzzles astronomers for more than a decade. We describe our new analysis of all radial velocity data currently available for this star, including both the most recent Keck data and the older but more numerous CORALIE measurements.

Here we pay a major attention to the task of optimal scheduling of the future observation of this system. Applying several optimality criteria, we demonstrate that in the forthcoming observational season of HD 82943 (the winter 2014/2015) rather promising time ranges can be found. Observations of the near future may give rather remarkable improvement of the orbital fit, but only if we choose their time carefully.
\end{abstract}

Keywords. stars: planetary systems - stars: individual: HD 82943 - techniques: radial velocity - methods: data analysis - methods: statistical

\section{Introduction}

This paper can be treated as an addition to our recent work (Baluev \& Beaugé 2014) devoted to a reanalysis of the radial velocity (RV) data for a unique multi-planet host star HD 82943. Here we only briefly summarize the most important of our previous results (Sect. 2), and present new ones, related to seeking the optimal observation dates for this star (Sect. 3).

\section{Main results of the RV data analysis}

In our work we used the entire set of the RV data currently available for HD 82943 in the public literature. These include the old CORALIE (Mayor et al. 2004) and the recent Keck (Tan et al. 2013) data. The Keck data were separated in two independent subsets that were acquired before and after a hardware upgrade. The primary results concerning our re-analysis of these data are described in (Baluev \& Beaugé 2014). Thus we do not duplicate this discussion here, except for a brief summary of the conclusions:

(a) The Keck and CORALIE data are not in a good agreement with each other: fitting the entire data set plainly leads to a severely unstable orbital configuration of the two major planets $b$ and $c$.

(b) One of the reasons for this mutual inconsistency is the likely presence of an additional systematic variation in the CORALIE (but not Keck) data with a period close 
Table 1. Prescribed observations scheduling goals for HD 82943.

\begin{tabular}{|l|r|c|}
\hline scheduling goal & critical parameters to refine & their number \\
\hline goal 1 & parameters of all three planets & 14 \\
goal 2 & common orbital inclination & 1 \\
goal 3 & parameters of the third planet & 4 \\
goal 4 & location relatively to the two-planet ACR (see text) & 3 \\
\hline
\end{tabular}

to a year. Likely, this variation appeared due to some imperfections of the spectrum processing algorithm used for CORALIE.

(c) After removal of the CORALIE annual variation, the RV data still contain a significant periodicity with a period of $\sim 1000 \mathrm{~d}$. We interpret this periodicity as a hint of the third planet that was previously suspected by Goździewski \& Konacki (2006) and Beaugé et al. (2008).

(d) An RV fit implying a stable planetary configuration can be obtained only by including both the third planet and the CORALIE annual term in the RV model. Without these terms, the nominal (best fitting) solution appears unstable due to an antialigned initial apsidal state of the two major planets, and forcing this configuration to be stable would infer an unsuitably large shift of the fit from its nominal position.

(e) The planets in the best fitting configuration lie near the three-planet resonance with the periods ratio $P_{c}: P_{b}: P_{d} \approx 1: 2: 5$. The motion of the first two planets is truly resonant (a libration) in the vicinity of an aligned Apsidal Corotation Resonance (ACR), while the orbit of the third planet is rather uncertain and its orbital evolution can represent a libration (i.e. a true resonance) as well as a circulation (not a genuine resonance). The structure of the dynamical space in the vicinity of the third planet's orbit is pretty complicated and involves resonant domains intervening with the non-resonant ones.

\section{Optimal planning of the future RV observations}

Clearly, the orbital and dynamical structure of the HD 82943 system is still rather uncertain. In view of this, it may be useful to apply some optimal planning routines, in order to predict the time segments in future in which the new RV observations would improve or knowledge about the system, as well as to identify the time ranges where the new observations would be almost useless.

We solve this task by means of the optimal planning approaches described in (Baluev 2008a). In this method we should select the entire set of the fitted parameters, or any their subset, or even a set of some other quantities expressed by smooth functions of the original parameters. Our goal is to find an optimal time for a new observation in the future, in order to achieve a maximum reduction of the uncertainties in the targeted quantities. Here we adopt the so-called D-optimality criterion, in which the "reduction" of a multi-dimensional uncertainty is treated in terms of the volumes ratio for the relevant uncertainty ellipsoids (or determinants of the relevant covariance matrices).

In this work we consider the three-planet fit with the eccentricity of the third planet always fixed at zero. Otherwise this eccentricity is ill determined and generates dramatic non-linearity effects, which are not desirable. Four sets of target quantities to refine were considered in this work, defining four scheduling "goals". These goals are described in Table 1. The goal 3 from this table is defined in a rather complicated manner. Its purpose is to refine our knowledge about the position of the dynamical system relatively to the ACR of the two major planets. This information is important for the long-term dynamics and the stability of the system (Beaugé et al. 2003). In this case the set of 
target quantities includes 4 partial derivatives of the averaged Hamiltonian of the two major planets. When all these derivatives vanish, we deal with an exact ACR, so by reducing their uncertainties we can improve our knowledge about whether the system is close to or far from the ACR. The derivatives are computed using the method from (Baluev 2008b).

The results of the computation are shown in Fig. 1, where we plot the graphs of a function that indicates how much the uncertainty of the target quantities would reduce, provided that we make a single observation at the time given in the abscissa. This relative reduction is normalized so that it corresponds to a single degree of freedom in the set of target quantities. The vertical fringes label the yearly seasons when the star can (darker bands) or cannot (white bands) be actually observed.

We can see series peaks in these plots, marking the position of optimal times. In Table 2 we show more details concerning these optimal times for the nearest three observation seasons.
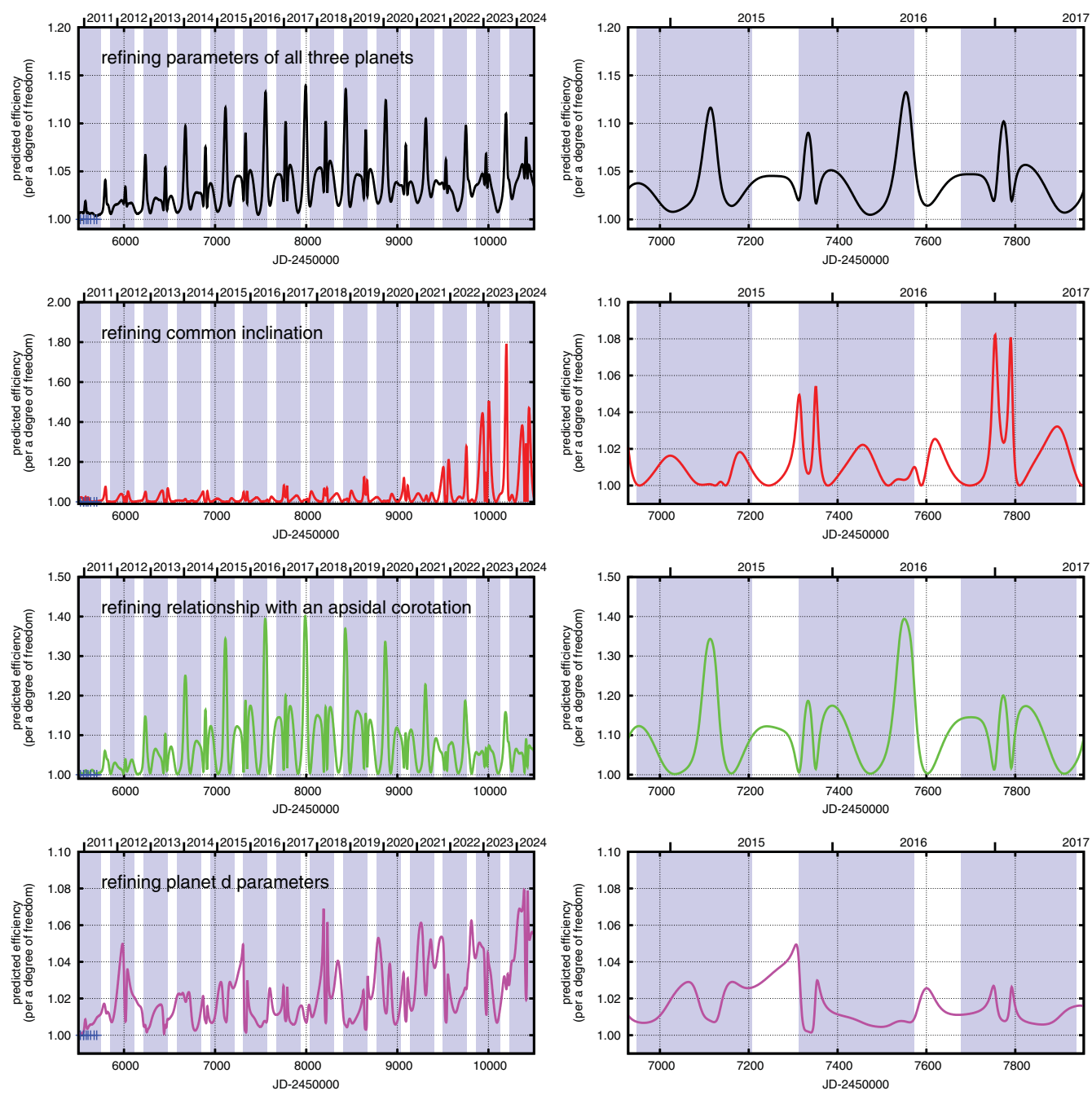

Figure 1. The predicted Keck observations efficiency for scheduling goals from Table 1. 
Table 2. Optimal observation dates for HD 82943.

\begin{tabular}{|c|c|c|c|c|c|c|c|}
\hline \multicolumn{2}{|c|}{ scheduling goal 1} & \multicolumn{2}{|c|}{ scheduling goal 2} & \multicolumn{2}{|c|}{ scheduling goal 3} & \multicolumn{2}{|c|}{ scheduling goal 4} \\
\hline JD-2450000 & \multicolumn{2}{|c|}{ max. eff. JD-2450000 } & \multicolumn{2}{|c|}{ max. eff. JD-2450000 } & \multicolumn{2}{|c|}{ max. eff. JD-2450000 } & max. eff. \\
\hline \multicolumn{8}{|c|}{ observational season of $2014 / 2015$} \\
\hline begin -6988 & 1.038 & $\mid 7024 \pm 27$ & 1.016 & begin -6985 & 1.123 & $7055 \pm 32$ & 1.029 \\
\hline $7113 \pm 15$ & 1.116 & $7182 \pm 17$ & 1.018 & $7113 \pm 17$ & 1.343 & 7140 - end & 1.029 \\
\hline 7193 - end & 1.035 & - & - & 7201 - end & 1.095 & - $\quad$ & - \\
\hline \multicolumn{8}{|c|}{ observational season of $2015 / 2016$} \\
\hline $7334 \pm 10$ & 1.090 & $7313 \pm 8$ & 1.049 & $\mid 7334 \pm 10$ & 1.187 & begin -7315 & 1.049 \\
\hline $7392 \pm 33$ & 1.051 & $7351 \pm 5$ & 1.054 & $7393 \pm 32$ & 1.175 & $7357 \pm 8$ & 1.030 \\
\hline $7553 \pm 16$ & 1.133 & $7454 \pm 26$ & 1.022 & $7551 \pm 21$ & 1.394 & - & - \\
\hline \multicolumn{8}{|c|}{ observational season of $2016 / 2017$} \\
\hline begin -7743 & 1.047 & $7755 \pm 7$ & 1.082 & begin -7739 & 1.145 & $7748 \pm 11$ & 1.027 \\
\hline $7773 \pm 10$ & 1.102 & $7790 \pm 5$ & 1.080 & $7773 \pm 10$ & 1.200 & $7793 \pm 6$ & 1.026 \\
\hline $7831 \pm 33$ & 1.057 & $7891 \pm 28$ & 1.032 & $7831 \pm 31$ & 1.174 & - & - \\
\hline
\end{tabular}

\section{Conclusions}

A few interesting matters can be noticed in Fig. 1 and Table 2:

(a) The peaks of the efficiency functions are rather narrow, meaning that allocating observation time randomly is not the best course of actions for HD 82943 .

(b) The task of refining the orbital inclination looks antagonistic to refining the most other parameters. But nonetheless the relevant optimal time ranges tend to stick together side-by-side.

(c) We have good chances to refine the accuracy of the usual planetary parameters by up to $30-40 \%$ in the forthcoming observing seasons. But the orbital inclination, which is only constrained thanks to the gravitational planet-planet perturbations, is an exception. It looks unrealistic to drastically improve the accuracy of this inclination before 2020s, when the orbital apsidal lines make a larger fraction of a secular revolution.

(d) The refining of the parameters of the third planet seems rather difficult both in the near and distant future. It seems that to reach this goal we should just patiently accumulate more and more observations.

The work was supported by the President of Russia grant for young scientists (MK-733.2014.2), by the Russian Foundation for Basic Research (project 14-02-92615 KO_a), and by the programme of the Presidium of Russian Academy of Sciences "Non-stationary phenomena in the objects of the Universe".

\section{References}

Baluev, R. V. 2008a, MNRAS, 389, 1375

Baluev, R. V. 2008b, Cel. Mech. Dyn. Astron., 102, 297

Baluev, R. V. \& Beaugé, C. 2014, MNRAS, 439, 673

Beaugé, C., Ferraz-Mello, S., \& Michtchenko, T. A. 2003, ApJ, 593, 1124

Beaugé, C., Giuppone, C., Ferraz-Mello, S., \& Michtchenko, T. A. 2008, MNRAS, 385, 2151

Goździewski, K. \& Konacki, M. 2006, ApJ, 647, 573

Mayor, M., Udry, S., Naef, D., Pepe, F., Queloz, D., Santos, N. C., \& Burnet, M. 2004, A\&A, 415,391

Tan, X., Payne, M. J., Lee, M. H., Ford, E. B., Howard, A. W., Johnson, J. A., Marcy, G. W., \& Wright, J. T. 2013, ApJ, 777, 101 